\title{
Supercrystallographic Reconstruction of 3D Nanorod Assembly with Collectively Anisotropic Upconversion Fluorescence
}

Kerong Deng, ${ }^{1, \ddagger}$ Xin Huang, ${ }^{2, ~}$ Yulian Liu, ${ }^{1}$ Lili Xu, ${ }^{3}$ Ruipeng Li, ${ }^{2}$ Ji Tang, ${ }^{4}$ Qun-li

Lei, ${ }^{5}$ Ran Ni, ${ }^{5}$ Chunxia Li, ${ }^{3, *}$ Yong Sheng Zhao, ${ }^{4, *}$ Hongwu Xu, ${ }^{6, *}$ Zhongwu Wang ${ }^{2, *}$ and Zewei Quan ${ }^{1,6, *}$

${ }^{1}$ Department of Chemistry, Southern University of Science and Technology (SUSTech), Shenzhen, Guangdong 518055, China

${ }^{2}$ Cornell High Energy Synchrotron Source, Cornell University, Ithaca, New York 14853, United States

${ }^{3}$ Institute of Molecular Sciences and Engineering, Shandong University, Qingdao 266237, China

${ }^{4}$ Key Laboratory of Photochemistry, Institute of Chemistry, Chinese Academy of Sciences, Beijing 100190, China

${ }^{5}$ School of Chemical and Biomedical Engineering, Nanyang Technological University, 62 Nanyang Drive, 637459, Singapore

${ }^{6}$ Earth and Environmental Sciences Division, Los Alamos National Laboratory, Los Alamos, New Mexico 87545, United States

${ }^{\ddagger}$ These authors contributed equally.

*Corresponding Authors. E-mail: cxli@sdu.edu.cn (C.L.); yszhao@iccas.ac.cn (Y.S.Z.); hxu@lanl.gov (H.X.); zw42@cornell.edu (Z.W.); quanzw@sustech.edu.cn (Z.Q.) 


\section{Experimental section}

Chemicals. $\mathrm{RECl}_{3} \cdot 6 \mathrm{H}_{2} \mathrm{O}(\mathrm{RE}=\mathrm{Y}, \mathrm{Yb}, \mathrm{Er}$; 99.99\%), oleic acid (OA; technical grade, 90\%), 1-octadecene (ODE; technical grade, 90\%) were purchased from Sigma Aldrich. $\mathrm{NH}_{4} \mathrm{~F}$ (A.R., 98\%) and $\mathrm{NaOH}$ (A.R., 97\%) were purchased from Aladdin. Methanol, hexane and ethanol were obtained from Shanghai Lingfeng chemical reagent company. All materials were used without further purification.

Nanocrystal synthesis. $\mathrm{NaYF}_{4}$ :Yb,Er NRs were prepared following the previous method with some modifications. ${ }^{1}$ Briefly, $1 \mathrm{mmol}$ of $\mathrm{RECl}_{3} \cdot 6 \mathrm{H}_{2} \mathrm{O}\left(\mathrm{RE}=80 \% \mathrm{Y}^{3+}+18 \% \mathrm{Yb}^{3+}+2 \% \mathrm{Er}^{3+}\right)$ was dissolved in $12 \mathrm{~mL}$ of $\mathrm{OA}$ and $30 \mathrm{~mL}$ of ODE at $150{ }^{\circ} \mathrm{C}$ and cooled to about $50{ }^{\circ} \mathrm{C}$. Then a methanolic solution $(10 \mathrm{~mL})$ containing $\mathrm{NaOH}(2.5 \mathrm{mmol})$ and $\mathrm{NH}_{4} \mathrm{~F}(4 \mathrm{mmol})$ was added. The mixture was degassed under vacuum for 30 min to remove residual methanol, water and oxygen, then heated to $320^{\circ} \mathrm{C}$ and maintained for $2 \mathrm{~h}$ under a nitrogen atmosphere. After the reaction was completed, the transparent yellowish nanocrystals were precipitated by adding ethanol and separated by centrifugation. The as-obtained nanocrystals were washed with ethanol two times, and redispersed in hexane as stock solution.

Assembly experiments. The growth of 3D NR-assembled superstructures was achieved from $\mathrm{NaYF}_{4}$ :Yb,Er NR suspensions in hexane at room temperature. Briefly, $5 \mathrm{~mL}$ of hexane solution of NRs (about $10 \mathrm{mg} / \mathrm{mL}$ ) was placed in a $30 \mathrm{~mL}$ of glass vial. The vial was then sealed with a plastic cap and left in an undisturbed environment, allowing the slow evaporation of the solvent. Large supercrystals were formed after complete solvent evaporation, and the assembly process took about two months. The NR supercrystal samples were preserved in the vial for further characterizations. The 2D NR assemblies were prepared by solvent evaporation-induced process. Briefly, a TEM Cu grid was placed on the silicon wafer with the size of $1 \mathrm{~cm} \times 1 \mathrm{~cm} .100 \mu \mathrm{L}$ of NR dispersion with an appropriate concentration was drop-casted onto the substrate, and the system was immediately sealed with a cover to slow down the evaporation. The 2D thin film was formed onto the substrate after complete evaporation of hexane.

Synchrotron X-ray scattering measurements. The X-ray scattering experiments were conducted at B1 station of Cornell High Energy Synchrotron Source (CHESS), Cornell University. The incident beam with a monochromatic wavelength of $0.4859 \AA$ was collimated to $100 \mu \mathrm{m}$ in diameter for the collection of both SAXS and WAXS patterns. The experimental apparatus was a custom-built setup described in detail by the previous work. ${ }^{2}$ For supercrystallography measurements, one piece of NR supercrystal was transferred onto a meshgrid (MiTeGen), and then mounted on a two-circle diffractometer. A series of SAXS and WAXS images were recorded upon rotation of the sample along one of its principal axis at an angular step of $1^{\circ}$ over the range of $180^{\circ}$. Both SAXS and WAXS signals were recorded by a MAR345 detector placed at $850 \mathrm{~mm}$ away from the sample. The sample-to-detector distance was calibrated by using a $\mathrm{CeO}_{2}$ standard sample of for these measurements. The 2D image calibration and preliminary analysis were conducted by the Fit2D software. Further analysis for 3D reciprocal space mapping was conducted 
with Matlab. The process to generate the 3D reciprocal space mapping was to convert the intensity from the raw image at certain pixel and rotation angle, I(px, py, $\Phi)$, into the intensity at certain position in the reciprocal space, I(Qx, Qy, Qz), including the correction of Ewald's sphere curvature and detector tilting.

Electron microscopy characterizations. Structural characterizations of NRs and assembled thin films were analyzed using HT7700 TEM (operated at $100 \mathrm{kV}$ ) and a FEI Tecnai F30 TEM (operated at $300 \mathrm{kV}$ ), including TEM images, high-resolution TEM images and SAED patterns. For SEM characterization, bulk supercrystals were carefully transferred to a silicon wafer. The SEM images were acquired from a ZEISS Merlin microscopy (operated at 1.0-2.0 kV).

Photoluminescence measurements. Photoluminescence measurements of NR supercrystal in this study were performed using a lab-built micro-photoluminescence setup. A continuous wave laser at $980 \mathrm{~nm}(5 \mathrm{~W})$ was used as the excitation source. Sample was transferred onto one glass slide and placed in a horizontally position on the sample stage. Then the laser spot $(\sim 10 \mu \mathrm{m}$ in diameter $)$ was locally focused on its surface by a microscopic objective. Upconversion emission was collected with the same object lens and monitored by a spectrometer (IsoPlane SCT 320), and then recorded with a CCD detector (PIX-1024BX). A short-pass filter at the spectrometer entrance was utilized to remove the excitation wavelength. In addition, excitation-dependent polarized upconversion luminescence was acquired by manually rotating a half-wave plate in an $8^{\circ}$ increment placed in the excitation path, while emission polarizer was maintained at vertical direction $\left(0^{\circ}\right)$. The photoluminescence data were carefully calibrated to eliminate the polarization dependence of the measurement setup. For the optical measurements of the supercrystals from additional direction, the sample was rotated vertically and kept on the substrate.

\section{References}

(1) Zhuang, Z.; Peng, Q.; Zhang, B.; Li, Y. Controllable synthesis of $\mathrm{Cu}_{2} \mathrm{~S}$ nanocrystals and their assembly into a superlattice. J. Am. Chem. Soc. 2008, 130, 10482-10483.

(2) Wang, Z.; Schliehe, C.; Bian, K.; Dale, D.; Bassett, W. A.; Hanrath, T.; Klinke, C.; Weller, H. Correlating superlattice polymorphs to internanoparticle distance, packing density, and surface lattice in assemblies of PbS nanoparticles. Nano Lett. 2013, 13, 1303-1311. 


\section{Supplementary figures and notes}

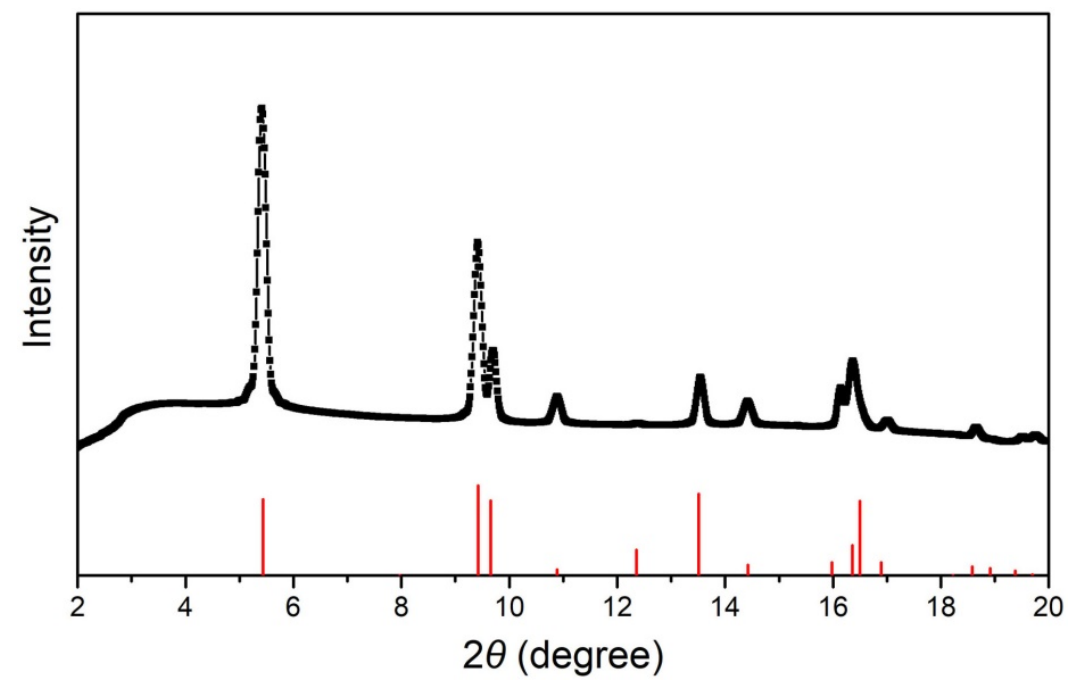

Figure S1. Integrated plot from synchrotron-based WAXS pattern of NR supercrystals, which corresponds to the calculated standard peaks from hexagonal phase $\mathrm{NaYF}_{4}$ (red stick, JCPDS card No. 16-0334). 

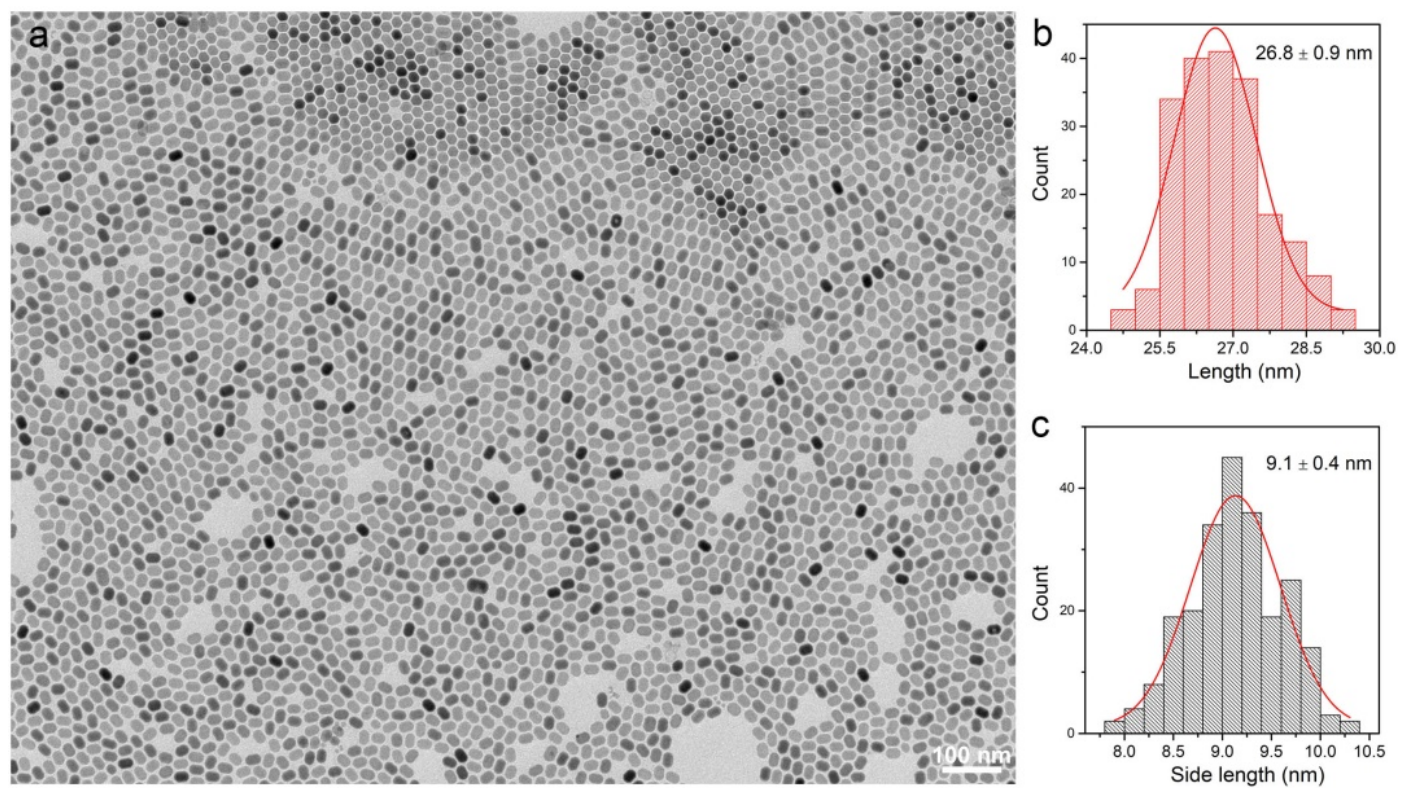

Figure S2. (a) Low-magnification TEM image of colloidal NRs. (b,c) The histograms of NR length (b) and side edge (c) distributions, respectively. 


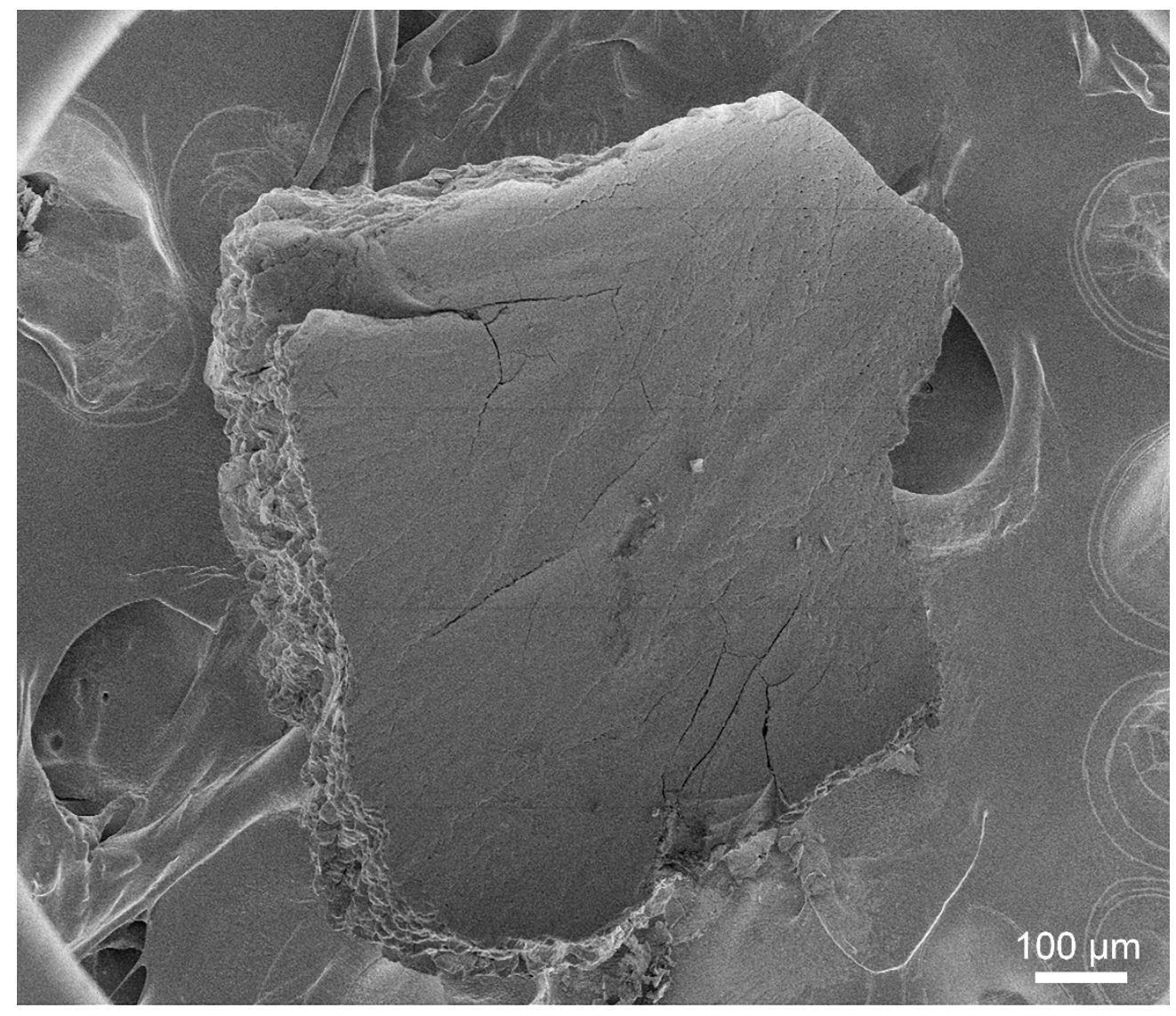

Figure S3. Typical SEM image for the as-obtained freestanding supercrystal. 


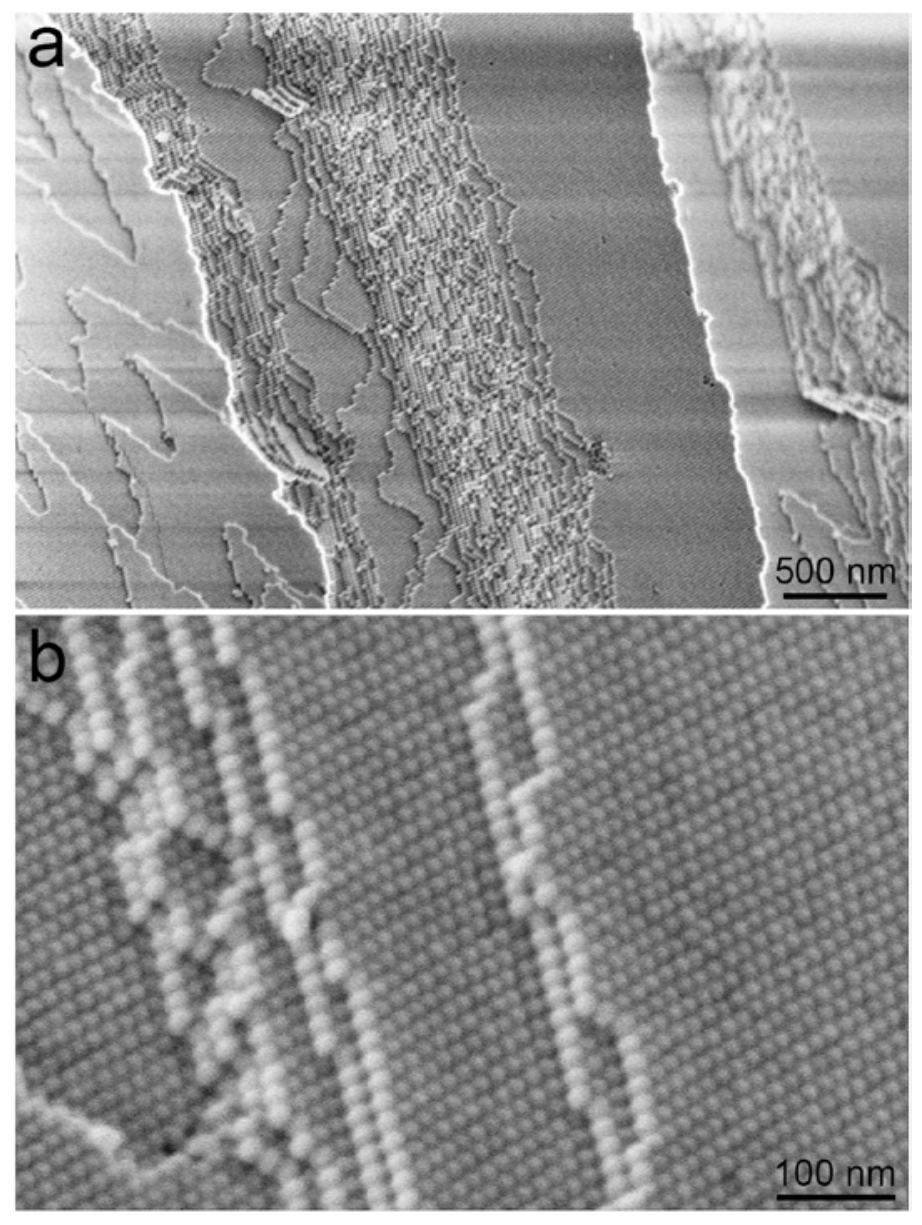

Figure S4. Additional SEM images of the supercrystal with different magnifications. 


\section{Reciprocal space mapping (RSM) analysis}

The fitting process is realized by calculating the scattering peaks from a structure model and then adjusting/orienting the structure model to let calculated peaks match the experimental scattering points in the 3D RSMs. Taking advantage of the RSMs for both SAXS and WAXS, any slice from the 3D reciprocal space could be selected and compared with the theoretical scattering patterns from the proposed model. For the NR supercrystal, the best fitted structure model is disclosed as two orthogonal sets of o-hcp SLs that share the [110] axis. Here three representative scattering planes are recognized to index one o-hcp SL, that are (-HHL) $)_{\mathrm{SL}},(\mathrm{HHL})_{\mathrm{SL}}$, and $(\mathrm{HK} 0)_{\mathrm{SL}}$ planes. To elucidate the complex structure with two orthogonal sets of identical o-hcp SLs, several sets of representative SAXS and WAXS patterns sliced from the 3D RSMs along various orientations are given in Figure 2 and Figures S5-S6.
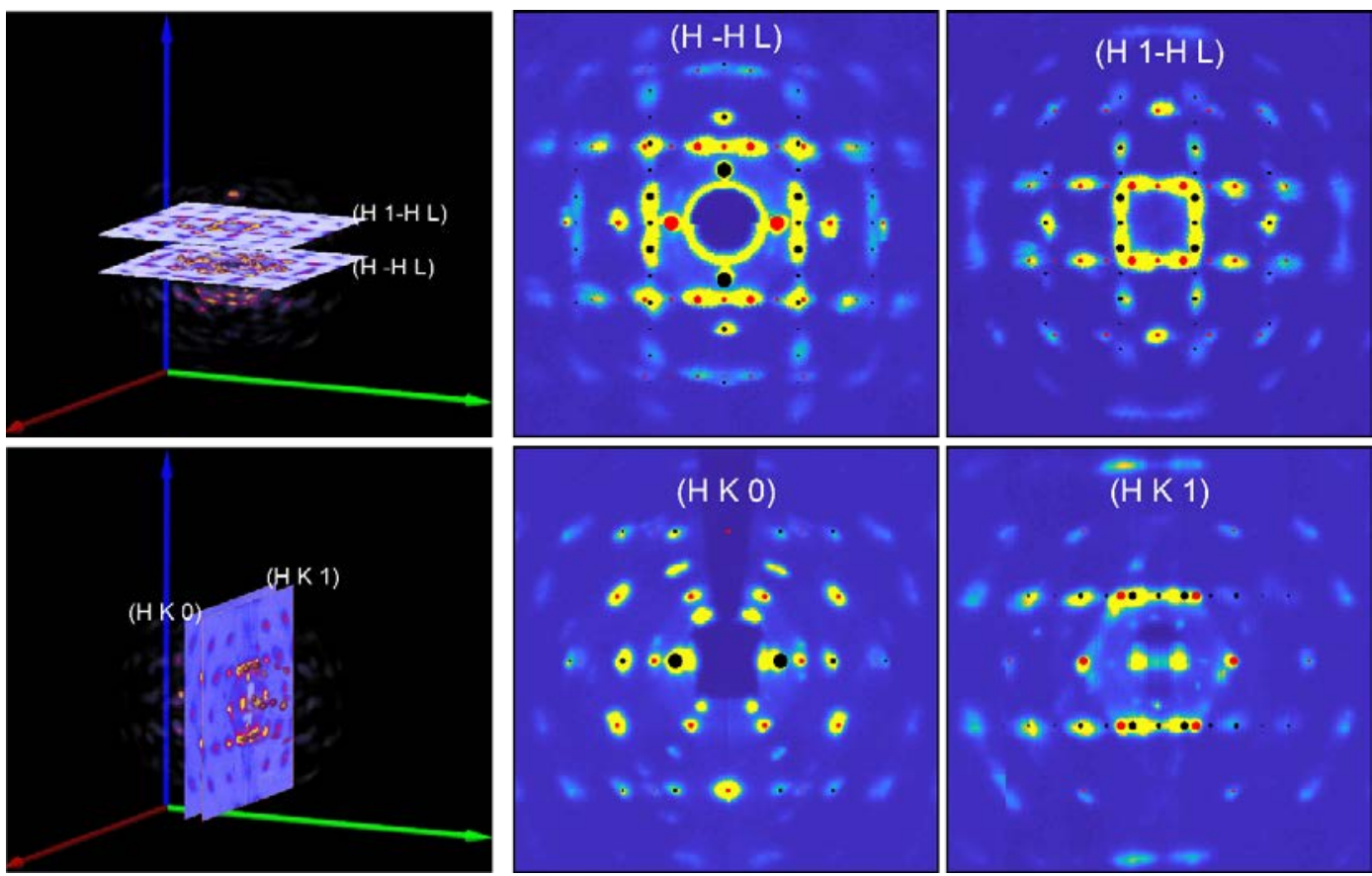

Figure S5. 2D images of the two representative SAXS patterns extracted from the 3D RSMs, respectively. (H 1-H L) plane means that all the scattering peaks (h k l) (red) follow the rules of $\mathrm{h}+\mathrm{k}=1$. (H K 1) means that all the scattering peaks (h k l) (red) following the rule of $\mathrm{l}=1$. 

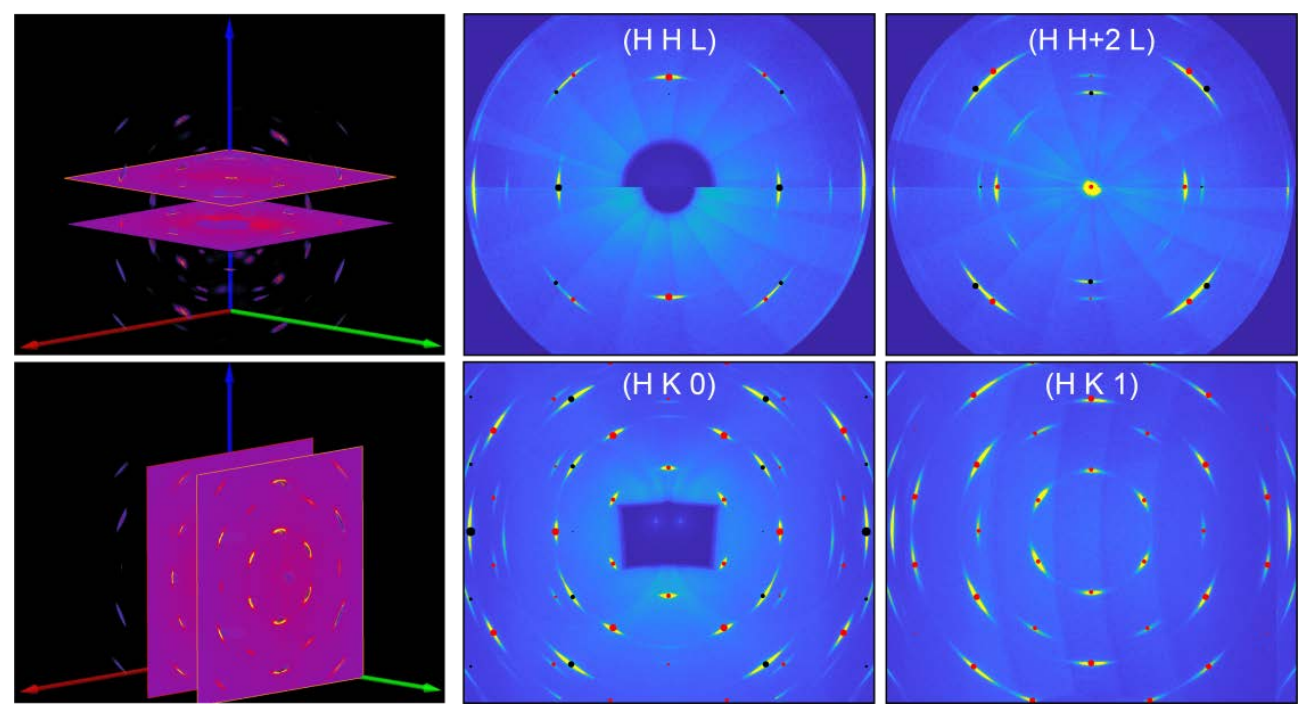

Figure S6. 2D images of the two representative WAXS patterns extracted from the 3D RSMs, respectively. ( $\mathrm{H} \mathrm{H}+2 \mathrm{~L}$ ) plane means that all the scattering peaks (h k l) (red) follow the rules of $\mathrm{k}-\mathrm{h}=2$. (H K 1) means that all the scattering peaks (h k l) (red) following the rule of l=1. 


\section{Quantitative analysis of two subdomains}

The ratio between SLa domain and SLb domain is quantified through the ratio of integrated intensity of representative scattering spots from SLa and SLb. The (-HHL) $)_{\mathrm{SL}}$ in 3D RSM in Figure 2a is selected for this purpose, because both SLs expose the same indexed spots in this plane. Figure S7 exhibits the horizontal reciprocal plane from the 3D RSM, where colored boxes mark the spots from SLa (red) and SLb (gray) for calculating their relative intensity ratio. The average intensity ratio of these spots from SLa and SLb in this supercrystal is calculated to be around 0.81 .

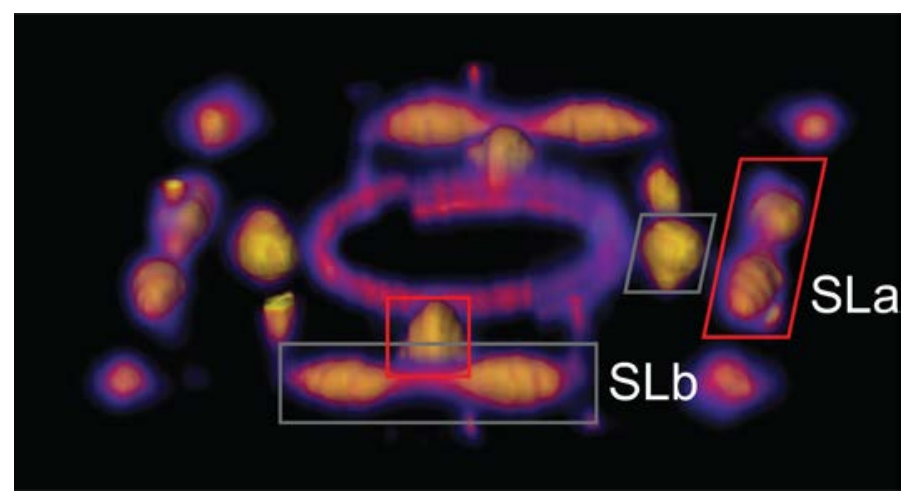

Figure S7. Selected region from 3D RSM for calculating the integrated intensity of representative scattering spots from SLa domain and SLb domain. The colored boxes are the integrated regions: red for SLa and gray for SLb. 

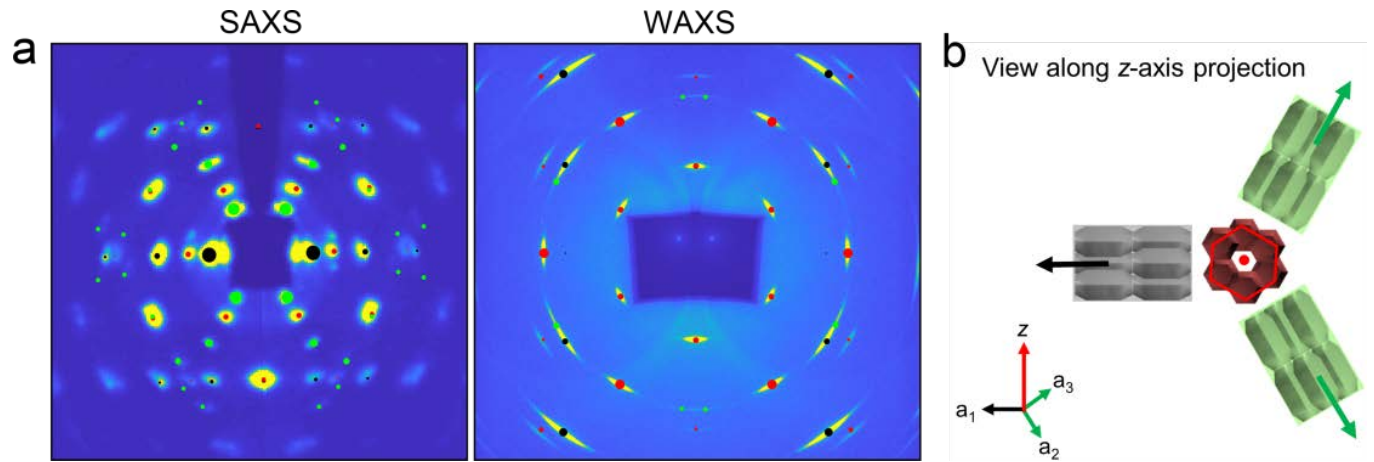

Figure S8. (a) 2D image of SAXS and WAXS patterns from the supercrystal at the $[001]_{\mathrm{SLa}} /[\overline{1} 10]_{\text {SLb }}$ projection in Figures $2 \mathrm{~g}$ and $2 \mathrm{i}$, showing the presence of several orientation-forbidden scattering spots. Green spots indicate the simulated scattering spots from the proposed model in (b). (b) Schematic representation for the main superstructures (light black and red blocks) with two additional SL domains (green blocks), leading to three domains in basal plane which are perpendicular to the vertical domain in the middle.

As shown in Figure S8a, several orientation-forbidden spots in the SAXS and WAXS patterns along the $[001]_{\mathrm{SLa}} /[\overline{1} 10]_{\mathrm{SLb}}$ projection can be found (green spots), mainly attributed to the presence of other subdomains. Apart from the two main o-hcp SLs with the orthogonal configuration (light black and red blocks), two additional sets of o-hcp SLs (green blocks) that also possess the same unit cell parameters, degenerate the in-plane orientation of light black one, and they orient in a trigonal-planar fashion in-plane. All domains behave in an identical fashion in the NR packing, but with different orientational directions. 

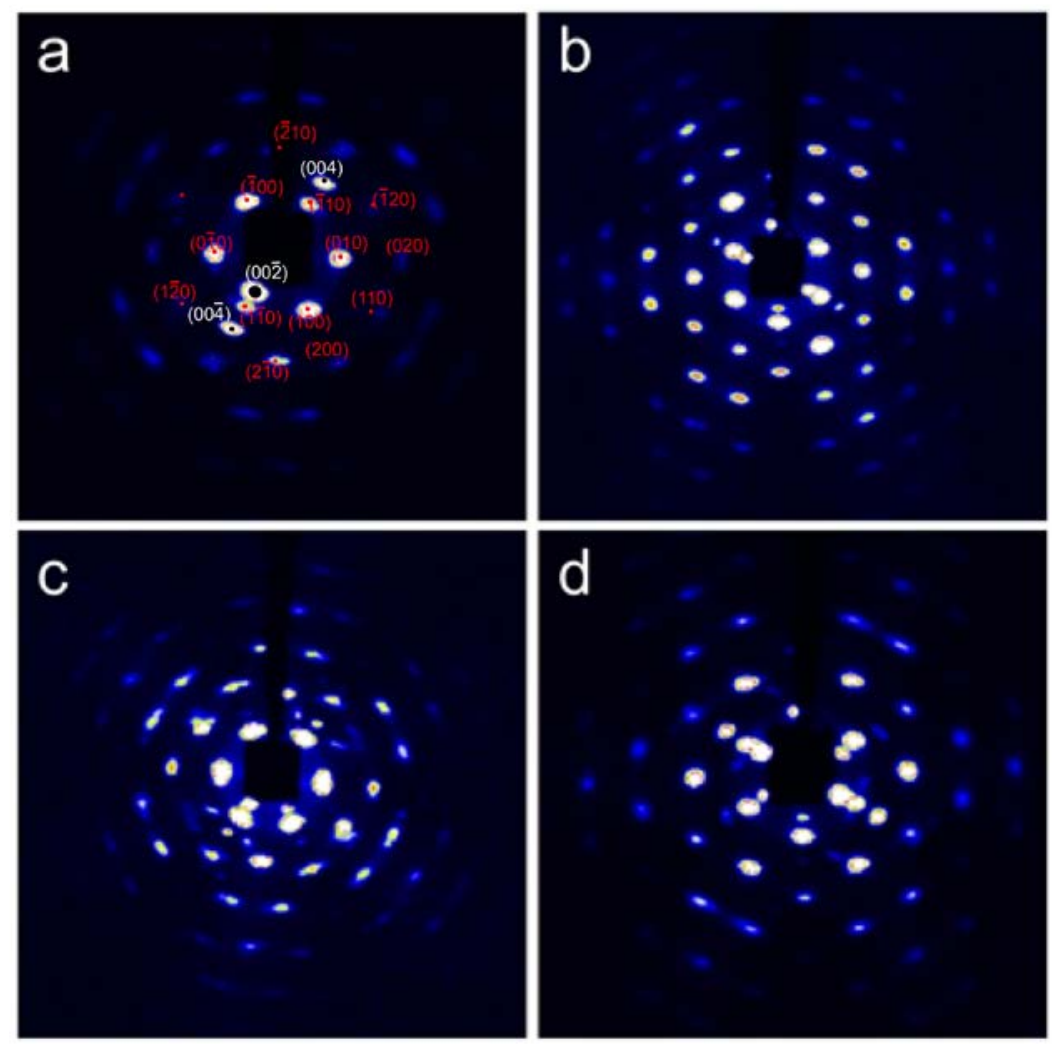

Figure S9. Additional SAXS patterns obtained from different pieces of the NR-based supercrystals.

For comparison, other SAXS patterns along the same $[001]_{\mathrm{SLa}} /[\overline{1} 10]_{\mathrm{SLb}}$ projection obtained from different pieces of the supercrystals are shown in Figure S9. It is clear that the observed orientation-forbidden spots appear randomly in these scattering patterns (for example, none of the orientation-forbidden spots is observed in Figure S9a), suggesting that it appears to be dependent on the thickness and size of the specimens. The results reveal the randomness of the additional minor subdomains inside these supercrystals, and further support the main structure of two orthogonal sets of o-hcp SLs. 

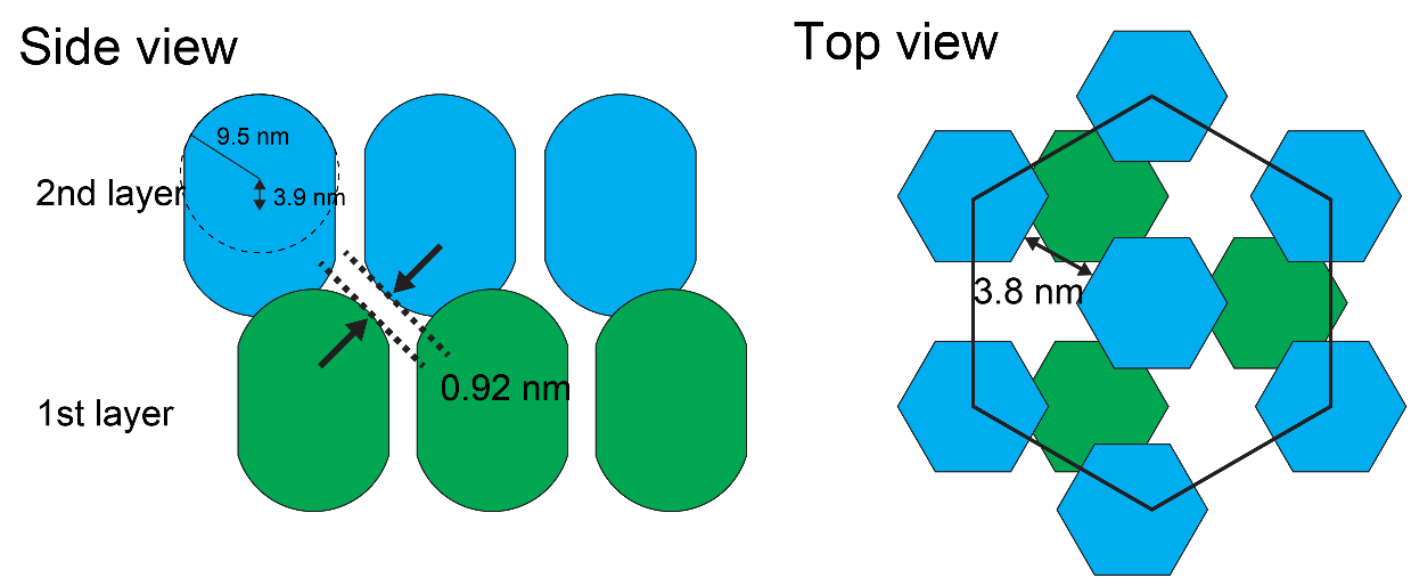

Figure S10. Schematic illustration of the relationship between NRs situated in two adjacent layers of the o-hcp SL. 


\section{Calculations on volume fraction}

The shape of NR is approximately estimated through the intersection of a capsule shape and a hexagonal prism.

The surface of the capsule shape is defined as:

$$
\begin{gathered}
x^{2}+y^{2}+(z-3.9)^{2}=9.5^{2}(\text { when } z>3.9 \mathrm{~nm}) \\
x^{2}+y^{2}+(z+3.9)^{2}=9.5^{2}(\text { when } z<-3.9 \mathrm{~nm}) \\
x^{2}+y^{2}=9.5^{2}(\text { when }-3.9 \mathrm{~nm}<z<3.9 \mathrm{~nm})
\end{gathered}
$$

The surface of the hexagonal prism shape is defined as:

$$
\begin{gathered}
x= \pm 9.1 \times \frac{\sqrt{3}}{2} \\
\cos (60) x-\sin (60) y= \pm 9.1 \times \frac{\sqrt{3}}{2} \\
\cos (60) x+\sin (60) y= \pm 9.1 \times \frac{\sqrt{3}}{2}
\end{gathered}
$$

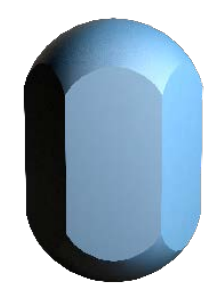

Therefore, the volume of the intersection (one NR) is calculated to be $V_{N C}=4.82 \times 10^{3} \mathrm{~nm}^{3}$. Since the volume of o-hcp unit cell is $V_{S L}=\frac{3 \sqrt{3}}{2} a^{2} c=4.83 \times 10^{4} \mathrm{~nm}^{3}$, the volume fraction of this o-hcp SL is $\frac{6 V_{N R}}{V_{S L}}=59.8 \%$.

By including the oleic acid ligands into the calculation, the volume of one NR ranges from $6.24 \times 10^{3} \mathrm{~nm}^{3}$ to $7.75 \times 10^{3} \mathrm{~nm}^{3}$, if the average length of bonded oleic acid on single NR is 0.5 to 1 times of its free length $(1.9 \mathrm{~nm})$. The volume fraction therefore ranges from $77 \%$ to $96 \%$. 


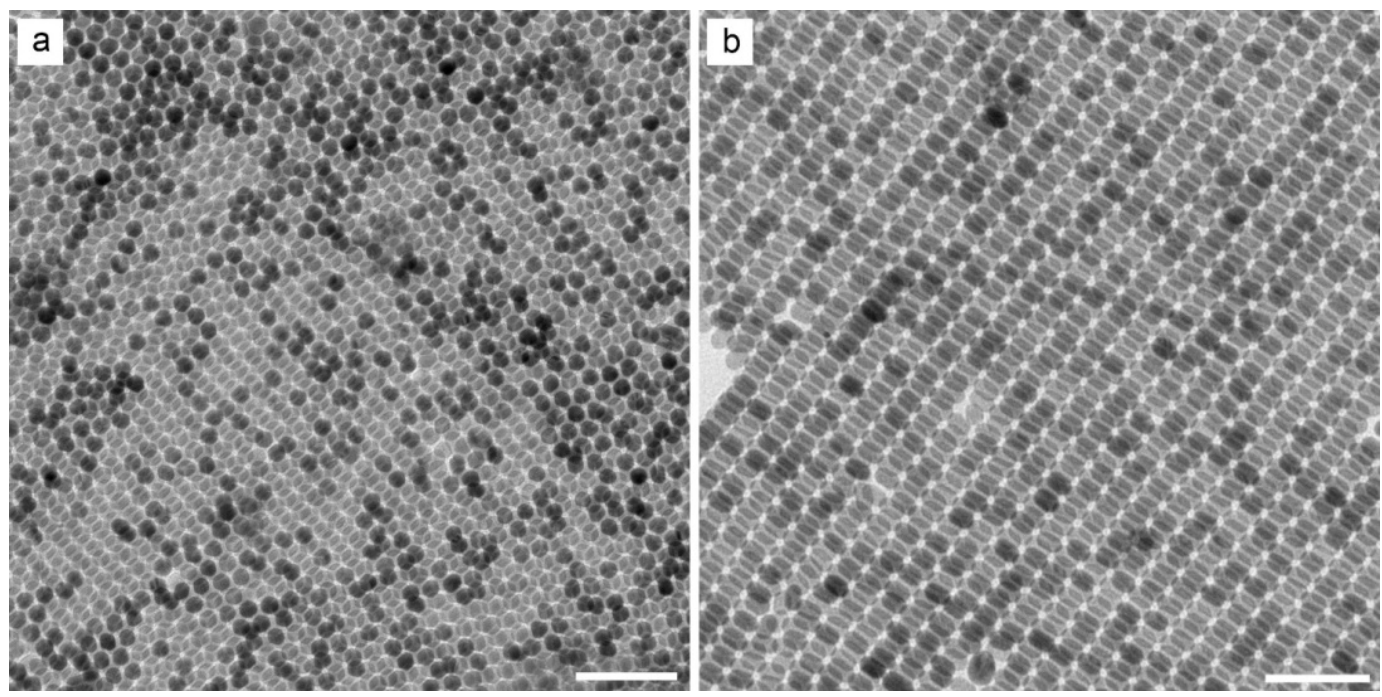

Figure S11. TEM images for double-layer NR SLs. Two types of packing behaviors are observed: NRs align their $c$ axis either normal (a) or parallel (b) to the substrate. Scale bars are $100 \mathrm{~nm}$.

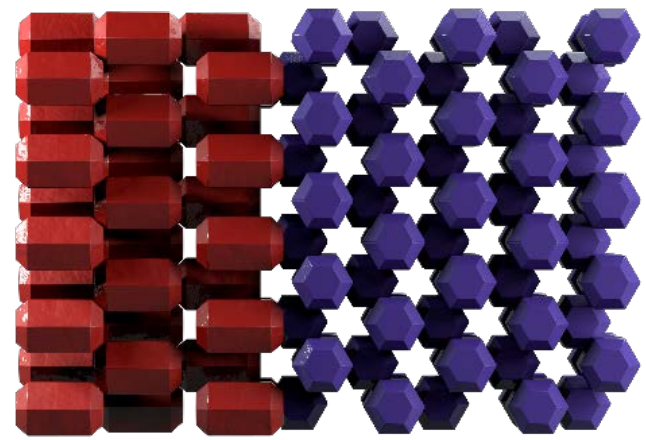

Figure S12. The schematic of the interface of two hexagonal stacking phases. 


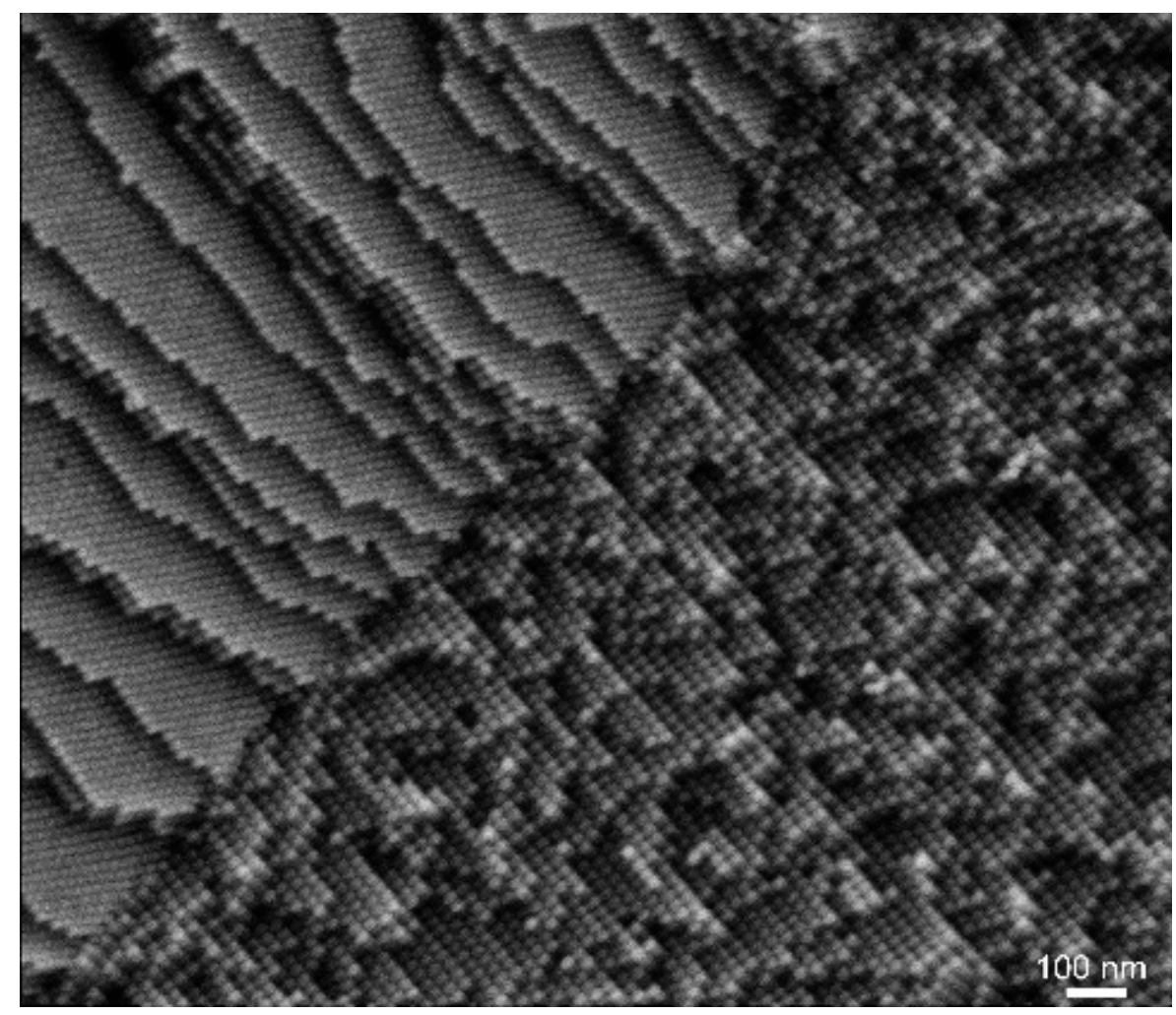

Figure S13. Typical SEM image of the grain boundary forming by two merging SL domains in the NR supercrystals. 


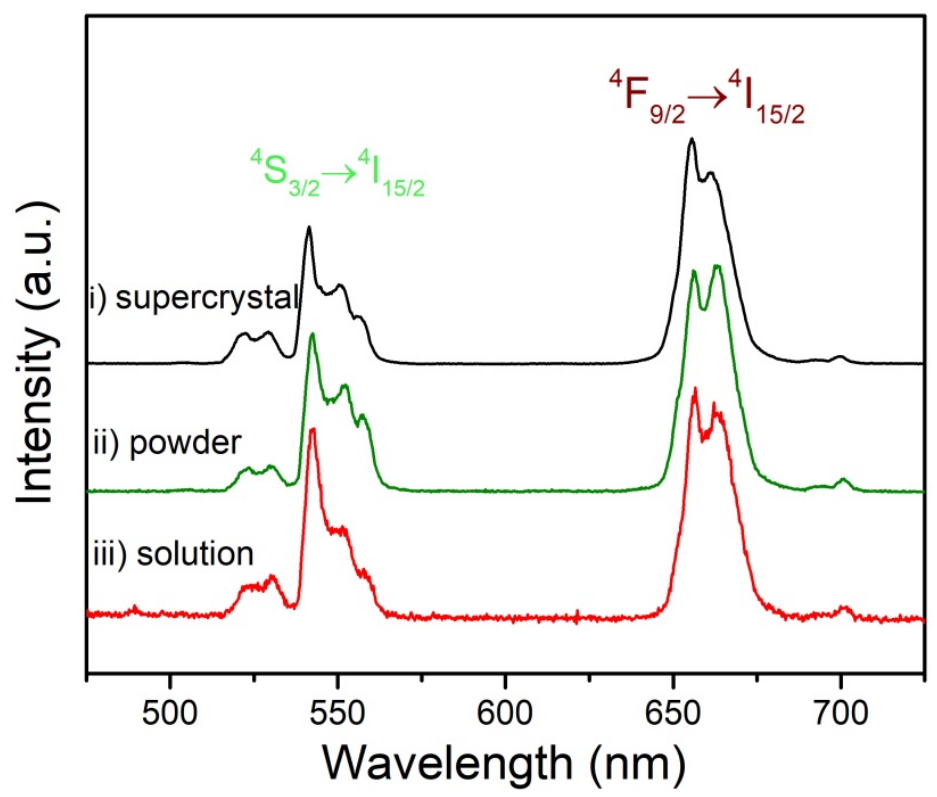

Figure S14. Photoluminescence spectra of NR supercrystal (black line), NRs powder (green line), and NR dispersion in hexane (red line) under the excitation of $980 \mathrm{~nm}$ laser, respectively. 


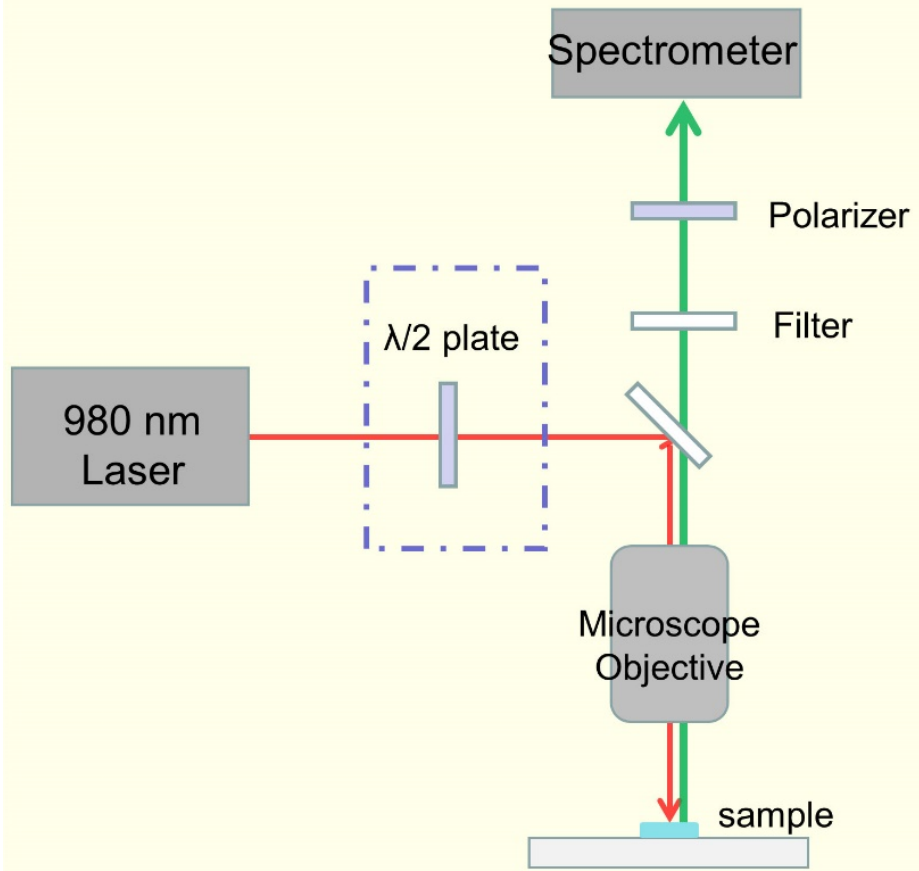

Figure S15. Schematic diagram of the experimental setup for the polarized photoluminescence measurements of the NR supercrystal sample. 


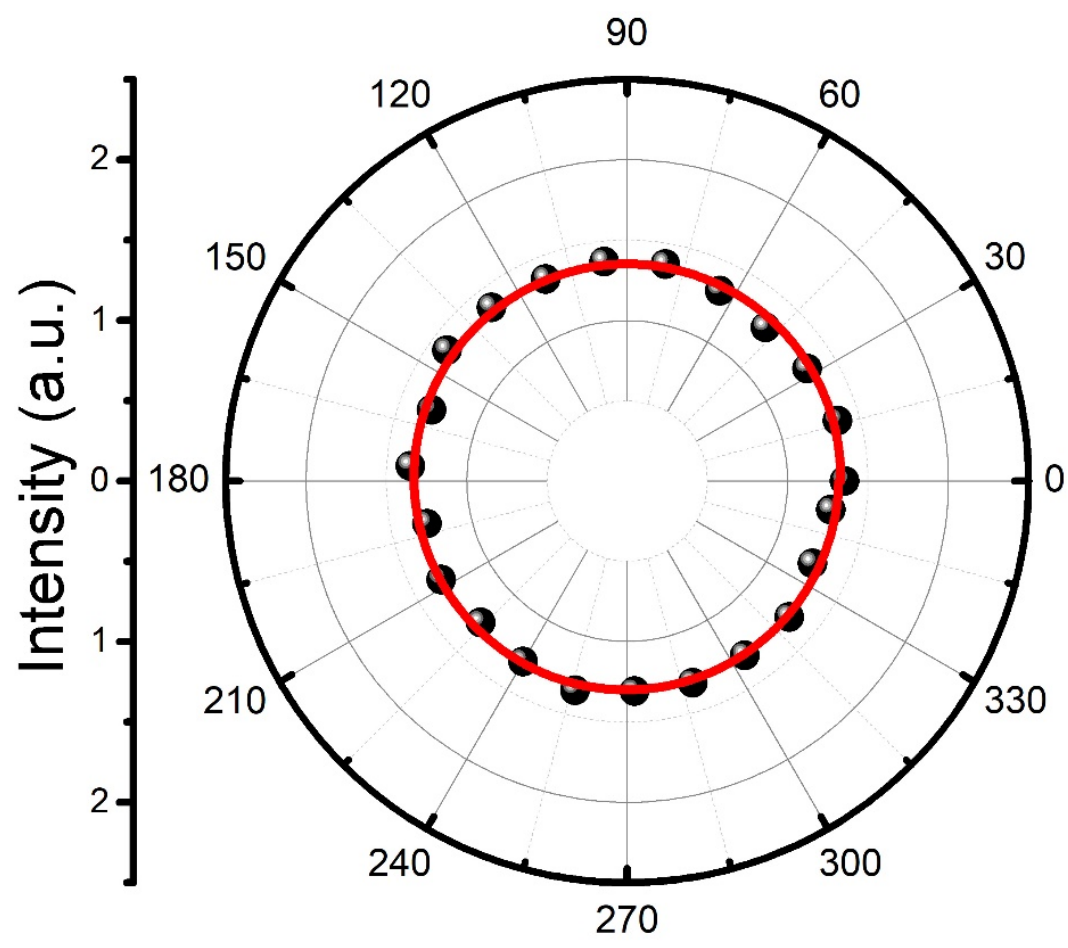

Figure S16. Polar plot of the emission intensity at $655 \mathrm{~nm}$ for disordered NRs powder as a function of the excitation polarization angle $\left(0 \sim 360^{\circ}\right)$. 


\section{Legends for supplementary videos}

Video S1: SAXS patterns of the NR supercrystals from the rotational SAXS measurement along the principal axis with a step angle of $1^{\circ}$.

Video S2: WAXS patterns of the NR supercrystals from the rotational WAXS measurement along the principal axis with a step angle of $1^{\circ}$.

Video S3: Corrected WAXS patterns of the NR supercrystals from the rotational WAXS measurement.

Video S4: 3D SAXS RSM constructed from the raw SAXS data.

Video S5: 3D WAXS RSM constructed from the corrected WAXS data. 